\title{
Bone loss and glucocorticoid therapy in patients with respiratory disease
}

\author{
Angela Fairney \\ Department of Endocrinology and Metabolic Medicine, Imperial College School of \\ Medicine, St Mary's Hospital, Paddington, London, UK
}

Introductory article

\section{Alendronate for the prevention and treatment of glucocorticoid-induced osteoporosis}

\author{
K G Saag, R Emkey, T J Schnitzer, J P Brown, F Hawkins, S Goemaere, G Thamsborg, U A Liberman, \\ P D Delmas, M-P Malice, M Czachur, A G Daifotis, for the Glucocorticoid-Induced Osteoporosis \\ Intervention Study Group
}

Background. Osteoporosis is a common complication of long-term glucocorticoid therapy for which there is no well-proved preventive or restorative treatment. Methods. We carried out two 48-week, randomized, placebo-controlled studies of two doses of alendronate in 477 men and women, 17-83 years of age, who were receiving glucocorticoid therapy. The primary end point was the difference in the mean percent change in lumbar-spine bone density from base line to week 48 between the groups. Secondary outcomes included changes in bone density of the hip, biochemical markers of bone turnover, and the incidence of new vertebral fractures. Results. The mean $( \pm S E)$ bone density of the lumbar $\stackrel{\Xi}{\Omega}$ spine increased by $2.1 \pm 0.3$ percent and $2.9 \pm 0.3$ percent, respectively, in the groups that received $5 \overrightarrow{\overrightarrow{0}}$ and $10 \mathrm{mg}$ of alendronate per day $(P<0.001)$ and decreased by $0.4 \pm 0.3$ percent in the placebo group. The femoral-neck bone density increased by $1.2 \pm 0.4$ percent and $1.0 \pm 0.4$ percent in the respective alendronate groups $(P<0.01)$ and decreased by $1.2 \pm 0.4$ percent in the placebo group $(P<0.01)$. The bone density of the trochanter and total body also increased significantly in the patients treated with 0 alendronate. There were proportionally fewer new vertebral fractures in the alendronate groups (overall $\underset{x}{\otimes}$ incidence, 2.3 percent) than in the placebo group (3.7 percent) (relative risk, 0.6; 95 percent confidence 3 interval, 0.1 to 4.4). Markers of bone turnover decreased significantly in the alendronate groups $(P<0.001)$. There were no differences in serious adverse effects among the three groups, but there was $₹$ a small increase in nonserious upper gastrointestinal effects in the group receiving $10 \mathrm{mg}$ of alendronate. 웅 Conclusions. Alendronate increases bone density in patients receiving glucocorticoid therapy. ( $N$ Engl $D$ J Med 1998;339:292-9).

Glucocorticoid induced bone loss is now a well recognise clinical problem. It may lead to osteoporotic fracture ${ }^{2}$ and occurs in patients being treated with glucocorticoids for different conditions such as rheumatoid arthritis $3^{3}$ asthma ${ }^{4}$ and polymyalgia rheumatica. Rapid bone loss may occur during the first year of treatment $t^{-}$and there is justified concern about the need for preventative strategies for patients starting on glucocorticoid treatment so that osteoporosis does not occur. However, the increased awareness of the need to diagnose and prevent osteoporosis in general the complex pathogenesis of steroid induced bone loss, the limited evidence to support current strategies for the prevention of steroid bone loss ${ }^{1}{ }^{11}$ and the pharmaceutical zeal to produce new agents and new applications of present one ${ }^{113}$ has led to a confused atmosphere for the practising clinician treating patients on glucocorticoids. There is a wide variety of advice available ${ }^{1 / 1}||_{10}^{19}$ but yncertainty remains about the precise way forward $2{ }^{22}$

Osteoporosis is defined as a progressive systemic 0 skeletal disease characterised by low bone mass and $\stackrel{\overrightarrow{0}}{\overrightarrow{0}}$ microarchitectural deterioration of bone tissue with a $\stackrel{\oplus}{9}$ consequent increase in bone fragility and susceptibility $\stackrel{\mathbb{2}}{2}$ to fracture 7 In the UK osteoporosis results in over 200000 fractures each year, at an annual cost to the o National Health Service of over $£ 940$ million, and more than one third of adult women will sustain one or more $\frac{\bar{Q}}{7}$

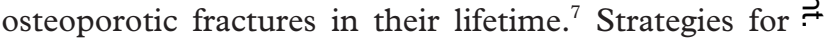
the prevention and identification of risk factors for osteoporosis are therefore important so that the burden of osteoporotic fractures can be reduced. Much attention has focused on risk factor frequency so that subjects at high risk of osteoporosis can be targeted for 
bone mass measurements and preventive treatment. The use of risk factor questionnaires has been limited in their success in identifying patients likely to develop fractures. However, it is now recognised that certain risk factors are more useful predictors of fracture than others ${ }^{23}$ These are reduced bone mineral density, history of a prior fracture after the age of 40 years, history of a fracture at the hip, wrist or vertebra in a first degree relative (family history), being in the lowest quartile in weight ( $\leqslant 57.8 \mathrm{~kg}$, thinness), and current cigarette smoking. Bone mass is accurately assessed by measurements of bone mineral density. A working group of the World Health Organisation has defined osteoporosis as a bone mineral density that is $2.5 \mathrm{SD}$ below the mean peak value in young adults ${ }^{24}$ This is known as the $\mathrm{T}$ score. Lesser degrees of bone loss are defined as osteopenia ( $\mathrm{T}$ score between $2.5 \mathrm{SD}$ and $1 \mathrm{SD}$ below the mean peak value in young adults). These limits are useful in making a diagnosis and also in assessing which patients are at risl ${ }^{25}$ Bone density is widely measured by dual energy $x$ ray absorptiometry (DXA) ${ }^{2}$ of the lumbar spine (particularly trabecular bone) and of the femoral neck (predominantly cortical bone). However, other methods involving assessment of the appendicular skeleton (peripheral DXA (pDXA) and ultrasound measurements of the calcaneus) are being developed for use in clinics and health centres. ${ }^{26}$

Bone remodelling is a complex process involving a number of cellular functions directed towards the coordinated resorption and formation of new bone. A sequence of events is initiated by local events so that osteoclastic activity produces bone resorption. This is followed by attraction, proliferation, and differentiation of osteoblast cells which lay down new bone and repair the resorption defects caused by resorbing osteoclasts. ${ }^{2}{ }^{28}$ The end point of bone remodelling is the maintenance of the bone mass. It is governed by systemic hormones and local factors including glucocorticoid and sex steroids. Bone loss may occur if the bone resorbed is not fully replaced due to overactivity of the osteoclast or underactivity of the osteoblast. The functions of osteoblasts and osteoclasts may be assessed by biochemical bone cell markers such as serum bone specific alkaline phosphatase, osteocalcin, and type I procollagen peptides (bone formation) and serum or urine pyridinoline crosslinks (bone resorption) ${ }^{29}$

\section{Extent of the problem}

It is generally accepted that moderate to high dose glucocorticoid therapy is associated with loss of bone and increased risk of fracture ${ }^{15}$ Some experts believe that at doses of more than $10 \mathrm{mg}$ prednisolone orally per day, all patients lose significant amounts of bone. ${ }^{17}$ This is not necessarily so as some Caucasian and AfroCaribbean patients often seem to be protected from bone loss and osteoporosis, even when receiving high doses of oral glucocorticoids. Also, not all postmenopausal women on chronic steroid and oestrogen treatment are osteoporoti ${ }^{30}$ and, in some instances, the glucocorticoid induced bone loss is superimposed on bone loss which is already increased because of the underlying disease process. Inflammation in polymyalgia rheumatica increases bone resorption and appears to have a more detrimental effect on bone than the glucocorticoid treatment ${ }^{31}$ This disorder is associated with high levels of cytokines such as interleukin $\phi^{32}$ which is known to stimulate osteoclast formation and prevents osteoclast apoptosis 33 Nevertheless, in many patients given high dose corticosteroids for many years (>10 mg prednisolone per day) bone loss is most rapid soon after starting treatment and occurs to a similar extent from the proximal femur and the lumbar spine ${ }^{6}$ A recent cross sectional study in general practice in Nottinghamshire was undertaken to $\frac{0}{2}$ determine the prevalence of continuous use of oral $\underset{x}{.}$ steroids in the general population and the extent to $\vec{F}$ which patients taking oralsferoids were taking treatment to prevent osteoporosis. ${ }^{34}$ Three hundred and three 등 patients were currently taking "continuous" oral gluco- $\overline{\bar{c}}$ corticoid treatment, representing $0.5 \%$ of the total $\underset{\otimes}{\otimes}$ population studied. The usual steroid prescribed was prednisolone (97\%) and the mean dose was $8.0 \mathrm{mg} /$ day. Nineteen per cent of the patients were receiving prednisolone for asthma and chronic obstructive airways $\vec{\omega}$ disease. Only 14\% (41) of the overall group of 303 o patients had received treatment for the prevention of $\overrightarrow{\vec{x}}$ osteoporosis. It is suggested that there may be at least or 250000 people in the UK taking continuous oral ster- i oids who need prophylaxis against osteoporosis.

However, in respiratory medicine many patients receive inhaled steroids, often with intermittent courses $N$ of oral prednisolone. There is concern about the effect 음 of this type of treatment and its potential for putting patients at risk of osteoporosis 35 The evidence from published studies has been confusing, partly because biochemical bone cell markers have been used at the $\stackrel{\infty}{+}$ end point as well as bone density. Even though inhaled $\vec{\bullet}$ beclomethasone at a dose of $2000 \mu \mathrm{g} /$ day produces a $\mathscr{\bigotimes}$ significant decline in serum osteocalcin after nine weeks of treatment ${ }^{36}$ and there is a decline in bone density in patients taking inhaled steroids only at more usual lower doses ${ }^{37}$ there is little evidence that long term treatment with inhaled steroids is asseciated with an increased risk $\frac{2}{\mathbb{Q}}$ of osteoporotic fractures $3^{340}$ It seems that it is a high daily dose of inhaled steroids, rather than the duration $\frac{0}{3}$ of the dose, that may adversely affect bone density. A comparison of the effect of fluticasone and budesonide on bone markers and bone density during one year of treatment did not show a decrease in bone density ${ }^{41}$ 으

Glucocorticoids produce hone loss by means of direct $\stackrel{0}{x}$ and indirect effects on bone ${ }^{17}$ Histomorphometric stud- $\frac{\dot{\sigma}}{3}$ ie ${ }^{42}$ indicate that glucocorticoids depress osteoblast function. In patients treated with glucocorticoids there $\frac{0}{3}$ is a greater than normal deficit in the quantity of bone replaced in each remodelling cycle. This leads to an enhanced rate of bone loss, which is more severe in $\frac{D}{0}$ parts of the skeleton with a high proportion of cancellous bone such as the vertebrae and ribs, where fractures $\sigma$ frequently occur. The low osteoblast activity is con- N firmed by reduced osteocalcin_levels in glucocorticoid $\mathbb{N}_{\mathrm{N}}$ dependent asthmatic patients. ${ }^{4}$ The possible reversibility of the effects of glucocorticoids on the osteoblast 6 is important from the clinical point of view. There is evidence from patients treated for Cushing's syndrome ? that the deleterious effects of glucocorticoids on the $\frac{T}{0}$ skeleton are reversible ${ }^{45}$ However, in practice if loss $\frac{0}{\mathbb{D}}$ of trabecular plates and microfractures has occurred within the bone, it is unlikely that a significant reversal $\stackrel{\mathbb{Q}}{9}$ of the effect can be expected on withdrawal of glucocorticoid treatment.

In addition, there are important indirect effects of glucocorticoids on bone ${ }^{17}$ These include a generalised defect in calcium transport, secondary hyper- + parathyroidism, and deficiency of anabolic hormones (table 1). As a result there is impaired gastrointestinal absorption of calcium, hypercalciuria, secondary hyperparathyroidism, deficiency of oestrogen and testosterone, as well as the direct effects on bone turnover. 


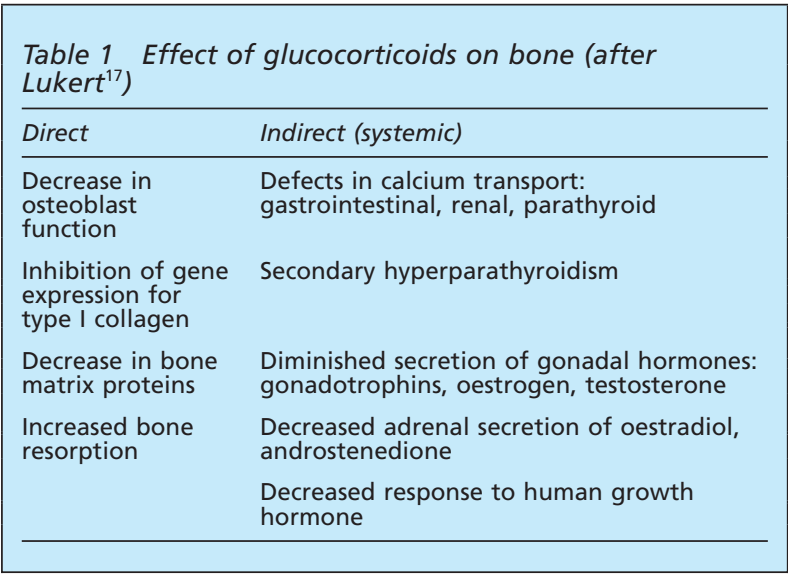

\section{Prevention by bisphosphonates}

PAPER BY SAAG ET A ${ }^{13}$

In order to prevent the skeletal side effects of glucocorticoid treatment there is much interest in developing strategies for use in clinical practice. The bisphosphonate drugs have been widely used as agents to prevent bone resorption ${ }^{46}$ and the paper by Saag and colleague ${ }^{13}$ for the Glucocorticoid Induced Osteoporosis Interventional Study Group reports the results of a randomised placebo controlled study of the amino bisphosphonate alendronate in 477 patients who were receiving glucocorticoid therapy. The patients studied were men and women of a wide age range (18-83 years) who were receiving their treatment for a variety of different disorders of which only up to $10 \%$ were for respiratory disorders. The multicentre study was conducted in two parts in parallel, one involving 232 patients at 15 centres in the USA and the other involving 328 patients at 22 centres in 15 other countries. The patients enrolled were receiving at least $7.5 \mathrm{mg}$ prednisolone or equivalent for different lengths of time $(<4$ months to $>12$ months) and were included in the study irrespective of baseline bone mineral density. The patients were randomly assigned to receive placebo or $5 \mathrm{mg}$ or $10 \mathrm{mg}$ alendronate. The treatment lasted for less than a year ( 48 weeks) and all patients received calcium and vitamin D supplements daily. There were a significant number of exclusion criteria but, of the 232 postmenopausal women included (49\%), 34\% were taking oestrogen replacement. One hundred and forty one men $(29.5 \%)$ were included in the study. The biochemical bone markers measured at the beginning of the study were within the reference range. In particular, the bone alkaline phosphatase (bALP) was not reduced as might be expected in response to glucocorticoid treatment. Unfortunately this study was not statistically powered to show the effect of alendronate on prevention of fractures.

This paper describes an international collaboration to study the use of alendronate for the prevention and treatment of glucocorticoid induced osteoporosis. Alendronate is known to be a very effective agent for the prevention and treatment of osteoporotic fracture ${ }^{4} 49$ so studies of the use of alendronate in glucocorticoid bone loss have therefore been eagerly awaited. There was an increase in mean lumbar spine bone density of $2.1 \%$ and $2.9 \%$ in response to doses of $5 \mathrm{mg}$ and $10 \mathrm{mg}$ alendronate, respectively $(\mathrm{p}<0.001)$. The corresponding values for the placebo group showed a decrease of $0.4 \%$. The increases in the femoral neck bone density were less remarkable and the biochemical bone marker responses decreased significantly in the alendronate treated patients $(\mathrm{p}<0.001)$.

In postmenopausal women under 60 years of age $5 \mathrm{mg}$ of alendronate given daily in order to prevent bone loss produced an increase in bone density at the lumbar 응 spine of $2.7 \%{ }^{47}$ These patients were not receiving $\stackrel{x}{x}$ hormone replacement therapy and the majority did not $\vec{F}$ have bone mineral density results in the osteoporotic $\frac{\text { ? }}{9}$ range. Saag et al reported that $5 \mathrm{mg}$ alendronate given $\frac{\mathrm{C}}{0}$ to postmenopausal women on glucocorticoid treatment $\overline{\bar{c}}$ who were receiving oestrogen replacement produced a $\vec{\nabla}$ $1.6 \%$ increase in lumbar spine density with similar results $(1.5 \%$ increase) following $10 \mathrm{mg}$ alendronate. क However, in the patients not receiving oestrogen the $\vec{\circ}$ postmenopausal women responded by an increase of $\vec{\overrightarrow{ }}$ $4 \%$ following $10 \mathrm{mg}$ alendronate. This suggests that $\stackrel{\omega}{\omega}$ women not on oestrogen replacement but receiving $\overrightarrow{\vec{x}}$ glucocorticoid therapy may respond more to alen- of dronate when lumbar spine bone density is used as the outcome parameter.

The duration of glucocorticoid therapy did not have any effect on the response to treatment, nor apparently of did the underlying disease state. However, there is $N$ concern about the minimal loss of lumbar spine bone mass in the patients in the placebo group, as would be $\vec{\nabla}$ expected in response to glucocorticoids. This suggests that the effect of alendronate in preventing bone loss $\frac{5}{\omega}$ may not have been very robustly tested in some patients. In the subgroup of patients with bullous skin diseases who were receiving high daily doses of glucocorticoids (mean dose $23.5 \mathrm{mg}$ prednisolone or equivalent) lumbar spine bone density in the patients in the placebo group decreased by $3 \%$ while in those treated with alendronate it increased by $2 \%$ from baseline, giving a total difference $\frac{2}{\Phi}$ of $5 \%$. Biochemical bone markers of bone turnover, bone formation, and bone resorption are known to $\vec{a}$ decrease in response to alendronate treatment in postmenopausal women 5 Also, bone turnover is increased in postmenopausal patients with osteoporosis. ${ }^{2}$ The effect of glucocorticoids on bone suppresses bone turnover ${ }^{10}$ especially by suppressing osteoblast activity. Approximately $50 \%$ of the patients treated with alen- $\underset{-}{-}$ dronate in the study by Saag et al underwent bone 3 . marker measurements. The type of patients (sex and $\dot{\delta}$ postmenopausal status) is not revealed. However, the response curves are related to reference values for pre- 음 menopausal women. There is evidence of increased bone resorption (urinary $\mathrm{N}$ telopeptides of type I collagen) at baseline and in the placebo group. However, the bone formation marker (serum bone specific alkaline $\sigma$ phosphatase) is within the reference range at baseline $\tilde{N}$ and was not suppressed, as might have been expected. In addition, the placebo values throughout the study did not decrease in response to glucocorticoid therapy. Serum osteocalcin (another bone formation marker used $\bar{\Phi}$ as an indicator of osteoblast activity) is known to decrease in concentration following beclomethasone treat- $\frac{0}{0}$ ment in postmenopausal asthmatic women. ${ }^{36}$ It is $\overrightarrow{\mathbb{D}}$ possible that, although bone specific alkaline phos- $\frac{\rho}{\mathbb{D}}$ phatase and osteocalcin are both osteoblast markers, $\stackrel{\Omega}{2}$ they may represent markers of different stages of osteoblast function ${ }^{53}$ and therefore respond to specific actions 8 of glucocorticoids on the osteoblast.

PREVIOUS BISPHOSPHONATE STUDIES

Intermittent treatment with etidronate has been shown to prevent corticosteroid induced bone loss in the lumbar spine $\left.5\right|^{56}$ No change in bone density occurred compared with placebo in the femoral neck and, when 
patients were treated with etidronate, bone loss at the

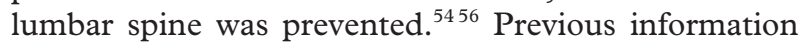
on the use of alendronate in glucocorticoid treated patients is very limited. In patients with sarcoidosis $5 \mathrm{mg}$ alendronate prevented radial bone loss of $4.5 \%$ in 12 months ${ }^{5 .}$ The improvement in bone density documented in these studies occurred within one year of treatment and it is possible that these changes may be related to bone remodelling transient ${ }^{58}$ It is therefore important that much longer studies assessing the effect of bisphosphonates on glucocorticoid bone loss are undertaken.

The structure-activity relationships of bisphosphonates and the subtle differences between the molecular mechanisms of action of etidronate and alendronate may be of interest when assessing the use of the drugs in preventing glucocorticoid induced bone loss. In vitro studies have shown alendronate to be significantly more potent than etidronat $\AA^{46}$ and, in postmenopausal osteoporosis, alendronate produces a greater increase in lumbar spine bone density than etidronate after three years of treatment ${ }^{4}||^{9}$ The limited data available on alendronate and etidronate do not show any evidence of difference in potency between these bisphosphonates when used to treat glucocorticoid induced bone loss. Bisphosphonates are pyrophosphate analogues with a strong affinity for mineralised tissues and are potent inhibitors of bone resorption 46 These compounds have a $\mathrm{P}-\mathrm{C}-\mathrm{P}$ backbone structure. The phosphorus atoms are linked to a geminal carbon having sites for two other groups (described as $R_{1}$ and $R_{2}$ ) which have enabled development of different analogues with different anti-bone resorptive potencies. The $R_{1}$ site is termed the head or hook and is the predominant determinant for hydroxyapatite binding. The $R_{2}$ site is termed the bone tail or bioactive moiety and is chemically modified to produce different compounds ${ }^{60}$ In etidronate the $R_{2}$ is a methyl group $\left(\mathrm{CH}_{3}\right)$ and in alendronate the $\mathrm{R}_{2}$ has a three-carbon side chain with a terminal amino group $\left(\left(\mathrm{CH}_{2}\right)_{3} \mathrm{NH}_{2}\right){ }^{61}$ The increased side chain increases lipophilicity which may permit better access to cell compartments. Also, the presence of an amino group in the side chain gives a many hundred times increase in inhibition of bone resorption in potency in vitro. ${ }^{60}$

Bisphosphonates act by inhibiting the activity of the osteoclasts leading to apoptosis. This may be by a direct action on the osteoclast or by stimulating the secretion of an inhibitor of osteoclast recruitment by the osteoblast.$^{4 .}$ Although the exact molecular targets have not yet been identified, there is some evidence that the amino bisphosphonates (such as alendronate) produce different molecular changes from the non-amino bisphosphonates such as etidronate and clodronate. Clodronate can be metabolised to a cytotoxic, non-hydrolysable analogue of ATP ${ }^{62}$ but the amino bisphosphonates are not metabolised in this way. The amino bisphosphonates cause apoptosis following inhibition of posttranslational prenylation of proteins such as Ras, as well as affecting the enzymes of the mevalonate pathway. 63 The bisphosphonates can therefore be grouped into those that are metabolised in cells and inhibit cytokine secretion from macrophages (such as etidronate and clodronate) and those that are not metabolised and enhance cytokine production (such as alendronate and pamidronate) ${ }^{64}$ This may be of particular importance in preventing bone loss in patients with inflammatory disease.

\section{Strategy for management}

How therefore should we manage our patients who are starting glucocorticoid treatment for respiratory disease? Before we can devise a strategy and consider the need for prophylactic treatment there are many variables that need to be recognised. For instance, is the patient at $\times$ risk of osteoporosis anyway because, for example, of a $\vec{F}$ family history of hip fracture? Does the patient already have undetected bone loss before the start of gluco- $\frac{\mathrm{C}}{0}$ corticoid treatment? Is the disease itself likely to cause $\overline{\bar{c}}$ bone loss because of bone resorption secondary to $\vec{\nabla}$ cytokines? Bone loss may occur very quickly in response to glucocorticoid treatment, or it may not occur at all. क At present we do not have the means to detect which $\vec{\circ}$ response is likely in a patient. Some ethnic groups may $\overrightarrow{\vec{\omega}}$ be more likely to develop steroid induced osteoporosis than others. If we use the drugs at present licensed for osteoporosis treatment such as hormone replacement of or bisphosphonates, how long should the treatment last? Will it give side effects? How should we monitor the

All patients starting oral corticosteroids therapy for 3 months or longer including patients on inhaled steroid if at a high dose should:

A. Be advised about lifestyle measures to prevent bone loss.

B. Have a bone density measurement of the lumbar spine and femoral neck at the beginning of treatment.

C. Be assessed for risk factors for osteoporosis (questionnaire) and have simple pathology tests to detect pre-existing risk factors for osteoporosis (LFTs, creatinine and electrolytes, bone profile, TSH, FBC and ESR testosterone (in men)).

A. Important life style measures to aid prevention of osteoporosis:

1. Maintain adequate dietary calcium and vitamin $\mathrm{D}$ intake (1500 $\mathrm{mg}$ calcium and 400 IU vitamin D per day).

2. Moderate alcohol intake.

3. Reduce cigarette smoking.

4. Daily weight bearing exercise.

5. Take lowest dose of glucocorticoid

B. If $B M D$ reduced: If $B M D$ normal: osteopenia ( $T-1$ to -2.5$)$ Treat risk factors if osteoporosis $(\mathrm{T}<-2.5) \quad$ possible

Start treatment

HRT

Bisphosphonates Ensure adequate calcium and vitamin $\mathrm{D}$

intake

Repeat BMD in 2 years 1 year

C. Risk factor assessment:

No. of cigarettes smoked per day.

Have you had a fracture if over 40 years of age?

Did one of your parents have a fracture?

Measurement of height and weight $\rightarrow$ BMI.

Dietary calcium intake.

Age.

Menopausal status.

BMD result.

Box 1 Suggested working schme for patients with respiratory disease starting glucocorticoid treatment. LFT=liver function test; disease starting glucocorticoid treatment. $L F T=$ liver function test;
$T S H=$ thyroid stimulating hormone; $F B C=$ full blood count; $E S R=$ $T S H=$ thyroid stimulating hormone; $F B C=$ full blood count; $E S R=$
erythrocyte sedimentation rate; $B M D=$ bone mineral density; $H R T=$ erythrocyte sedimentation rate;
hormone replacement therapy. 


\section{LEARNING POINTS}

\section{* Bone loss leading to osteoporotic fractures is a significant consequence of glucocorticoid treatment of respiratory disease.}

* Bone loss occurs primarily in patients receiving oral glucocorticoids which directly suppress osteoblast function.

\section{* Not all patients receiving glucocorticoid treatment develop bone loss.}

* Before the start of glucocorticoid treatment patients should be assessed for potential bone loss by bone densitometry and risk factor assessment.

* Bone loss can be prevented and treated by life style measures, hormone replacement, bisphosphonates, or vitamin $\mathrm{D}$ and calcium.

effect of treatment and is the cost justified? Are there other steroid like drugs that can be used that are bone sparing? Long term evidence for the prevention of glucocorticoid bone loss is not available and therefore a well founded strategy is difficult to formulate. A simple working scheme is proposed in box 1 .

Eastell and colleague ${ }^{10}$ have produced a very comprehensive review of the management of glucocorticoid induced osteoporosis. In particular, it provides well documented references to trials of the bisphosphonates and other agents for the prevention of steroid induced bone loss. However, it may not be necessary for all patients starting on oral glucocorticoid treatment for six months or more to have preventative therapy. More information is required about the dose of oral corticosteroid that causes bone loss. There is also some evidence that vertebral fractures may occur at a lower bone density in glucocorticoid treated patients than in those with other types of bone loss 65

1 Gennari C. Glucocorticoid induced osteoporosis. Clin Endocrinol 1994 41:273-4.

2 Adinoff AD, Hollister JR. Steroid induced fractures and bone loss in asthma. N Engl F Med 1983;309:265-8.

3 Spector TD, Hall GM, McCloskey EV, et al. Risk of vertebral fracture in women with rheumatoid arthritis. BMF 1993;306:558

4 Reid DM, Nicoll JJ, Smith MA, et al. Corticosteroids and bone mass in asthma: comparisons with rheumatoid arthritis and polymyalgia in asthma: comparisons with rheum.
rheumatica. $B M Y 1986 ; 293: 1463-6$.

5 Pearce G, Ryan PFJ, Delmas PD, et al. The deleterious effects of low dose corticosteroids on bone density in patients with polymyalgia rheumatica. Br 7 Rheumatol 1998;37:292-9.

6 Sambrook P, Birmingham J, Kempler S, et al. Corticosteroid effects on proximal femur bone loss. F Bone Mineral Res 1990;5:1211-6.

7 Royal College of Physicians. Osteoporosis, clinical guidelines for prevention and treatment. London: Royal College of Physicians, 1999.

8 Department of Health. Quick reference primary care guide on the prevention and treatment of osteoporosis. London: Department of Health, 1998

9 Lukert BP, Kream BE. Clinical and basic aspects of glucocorticoid action in bone. In: Bilezikian JP, Raisz LG, Rodan GA, eds. Principles of bone biology. London: Academic Press, 1996: 533-48.

10 Eastell R, Reid DM, Compston J, et al. A UK Consensus Group on management of glucocorticoid-induced osteoporosis: an update. $\mathcal{F}$ Intern Med 1998;244:271-92.

11 National Osteoporosis Society. Guidance on the prevention and management of corticosteroid induced osteoporosis. Bath, UK: National Osteoporosis Society, 1998

12 Struys A, Snelder AA, Mulder H. Cyclical etidronate reverses bone loss of the spine and proximal femur in patients with established corticosteroid induced osteoporosis. Am 7 Med 1995;99:235-42.

13 Saag KG, Enikey R, Schnitzer TJ, et al. Alendronate for the prevention and treatment of glucocorticoid induced osteoporosis. $N$ Engl f Med 1998;339:292-9.

14 Compston JE, Andran M, Avouac B, et al. Recommendations for the registration of agents in the prevention and treatment of glucocorticoid induced osteoporosis. Calcif Tissue Int 1996;59:323-7.

15 American College of Rheumatology Task Force on Osteoporosis Guidelines. Recommendations for the prevention and treatment of glucocorticoid induced osteoporosis. Arthritis Rheum 1996;39:1791-801.

16 Scientific Advisory Board, Osteoporosis Society of Canada. Clinical practice guidelines for the diagnosis and management of osteoporosis. Can Med Assoc F 1996;155:1113-33.

17 Lukert B. Glucocorticoid-induced osteoporosis. In: Marcus R, Feldman D, Kelsey J, eds. Osteoporosis. London: Academic Press, 1996: 801-20.

18 Cowan S, Morin S, Ernst P. Bisphosphonates and glucocorticoidinduced osteoporosis: implications for patients with respiratory diseases. Thorax 1998;53:331-2.

19 Tobias JH. Management of steroid-induced osteoporosis: what is the current state of play? Rheumatology 1999;38:198-201
20 Reid DM. Corticoid induced osteoporosis: guidelines for prevention are they useful? Br f Rheumatol 1997;36:1035-6.

21 Stevenson JC. Management of corticosteroid-induced osteoporosis. Lancet 1998;352:1327-8.

22 Seeman E, Ryan PJ. Bone turnover and bone mass in polymyalgia $\infty$ rheumatica Rh PJ Bology 1999:38:386.

23 Osteoporosis: review of the evidence for prevention, diagnosis and of treatment and cost-effectiveness analysis. Osteoporosis Int 1998;8 (Suppl $N$

24 World Health Organisation. Assessment of fracture risk and its application to screening for postmenopausal osteoporosis. Technical Report Series 843. Geneva: WHO, 1994.

25 Kanis JA, Melton JL, Christiansen C, et al. The diagnosis of osteoporosis. $\mathcal{J}$ Bone Mineral Res 1994;9:1137-41.

26 Blake GM, Wahner HW, Fogelman I. The evaluation of osteoporosis: dual $\stackrel{\infty}{\longrightarrow}$ energy $x$-ray absorptiometry and ultrasound in clinical practice. 2 nd ed.

27 Canalis E. Regulation of bone remodelling. In: Favus MJ, ed. Primer on the metabolic bone diseases and disorders of mineral metabolism. 3rd ed. Philadelphia: Lippincott-Raven, 1996: 29-34.

28 Munday GR. Bone remodelling and its disorders. UK: Martin Dunitz, 1995.

29 Calvo MS, Eyre DR, Gundberg CM. Molecular basis and clinical application of biological markers of bone turnover. Endocrine Rev 1996;17:333-68.

30 Thompson JM, Modin GW, Arnaud CD, et al. Not all postmenopausal $\stackrel{\mathbb{\Omega}}{\varrho}$ women on chronic steroid and oestrogen treatment are osteoporotic: predictors of bone mineral density. Calcif Tissue Int 1997;61:377-81.

31 Dolan AL, Moniz C, Dasgupta B, et al. Effects of inflammation and treatment on bone turnover and bone mass in polymyalgia rheumatica. Arthritis Rheum 1997;40:2022-9.

32 Dasgupta B, Panayi GS. Interleukin 6 in serum of patients with polymyalgia rheumatica and giant cell arteritis. Br f Rheumatol 1990;29: 456

33 Boyce $\mathrm{BF}$, Hughes DE, Wright KR, et al. Recent advances in bone biology provide insight into the pathogenesis of bone diseases. Lab $\times$ Invest 1999;79:83-94.

34 Walsh LJ, Wong CA, Pringle M, et al. Use of oral corticosteroids in the community and the prevention of secondary osteoporosis: a cross sectional study. BMF 1996;313:344-6.

35 Barnes PJ. Inhaled glucocorticoids for asthma. N Engl f Med 1995;332: $868-75$.

36 Puolijoki H, Liippo K, Herrala J, et al. Inhaled beclomethasone decreases serum osteocalcin in postmenopausal asthmatic women. Bone 1992; 13:285-8.

37 Packe GE, Douglas JG, McDonald AF, et al. Bone density in asthmatic patients taking high dose inhaled beclomethasone dipropionate and $\mathrm{N}$ patients taking high dose inhaled beclomethasone dipropion

38 Kerstiens HAM, Postma DS, van Doormaal JJ, et al. Effects of short term and long term treatment with inhaled corticosteroids on bone metabolism in patients with airways obstruction. Thorax 1994;49: స్ 652-6.

39 Herrala J, Puolijki H, Impivaara O, et al. Bone mineral density in asthmatic women on high dose inhaled beclomethasone dipropionate. $\bullet$ Bone 1994;15:621-3.

40 Toogood JH, Barkerville JC, Markov AE, et al. Bone mineral density and the risk of fracture in patients receiving long term inhaled steroid therapy for asthma. F Allergy Clin Immunol 1995;96:157-66.

41 Hughes JA, Conry BG, Male SM, et al. One year prospective open study of the effect of high dose inhaled steroids fluticasone propionate and budesonide on bone markers and bone mineral density. Thorax and budesonide

42 Dempster DW. Bone histomorphometry in glucocorticoid induced os- 2 teoporosis. $尹$ Bone Mineral Res 1989;4:137-41.

43 Reid IR, Chapman GE, Fraser TRC, et al. Low serum osteocalcin levels in glucocorticoid treated asthmatics. I Clin Endocrinol Metab 1986; 62:379-83.

44 Meunier PJ, Dempster DW, Edouard D, et al. Bone histomorphometry in corticosteroid induced osteoporosis and Cushings syndrome. Adv
Exp Med Biol 1984;171:191-200.

45 Pocock NA, Eisman JA, Dunstan CR, et al. Recovery from steroidinduced osteoporosis. Ann Intern Med 1987;107:319-23.

46 Fleisch H. Bisphosphonates in bone disease: from the laboratory to the patient. 3rd ed. Parthenon Publishing, 1997.

47 Hosking D, Chilvers CED, Christiansen C, et al. Prevention of bone loss with alendronate in postmenopausal women under 60 years of age. $N$ Engl f Med 1998;338:485-92.

48 Liberman UA, Weiss SR, Broll J, et al. Effect of oral alendronate on . 
bone mineral density and the incidence of fractures in postmenopausal osteoporosis. N Engl f Med 1995;333:1437-43.

49 Black DM, Cummings SR, Karpf DB, et al. Randomised trial of effect of alendronate on risk of fracture in women with existing vertebral fractures. Lancet 1996;348:1535-47.

50 Kyd PA, DeVooght K, Kerkhoff F, et al. Clinical usefulness of bone alkaline phosphatase in osteoporosis. Ann Clin Biochem 1998;35 $717-25$

51 Kyd PA, DeVooght K, Kerkhoff F, et al. Clinical usefulness of biochemical resorption markers in osteoporosis. Ann Clin Biochem 1999; 36 (in press).

52 Garnero P, Shih WJ, Gineyts E, et al. Comparison of new biochemical markers of bone turnover in late postmenopausal osteoporotic women in response to alendronate treatment. 7 Clin Endocrinol Metab 1994; 79:1693-700.

53 Stein GS, Lian JB. Molecular mechanisms mediating proliferation differentiation interrelationships during progressive development of the osteoblast phenotype. Endocrine Rev 1993;14:424-42.

54 Adachi JD, Bensen WG, Brown J, et al. Intermittent etidronate therapy to prevent corticosteroid induced osteoporosis. N Engl f Med 1997 337:383-7.

55 Pitt P, Li F, Todd P, et al. A double blind placebo controlled study to determine the effects of intermittent cyclical etidronate on bon mineral density in patients on long term oral corticosteroid treatment. Thorax 1998;53:351-6.

56 Roux C, Oriente P, Laan R, et al. Randomised trial of effect of cyclical etidronate in the prevention of corticosteroid induced bone loss. 7 Clin Endocrinol Metab 1998;83:1128-33.

57 Gonnelli S, Rottoli P, Cepollaro C, et al. Prevention of corticosteroid induced osteoporosis with alendronate in sarcoid patients. Calcif Tissue Int 1997;61:382-5.

58 Heaney RP. The bone remodelling transient: implications for the interpretation of clinical studies of bone mass change. $\mathcal{F}$ Bone Mineral Res 1994:9:1515-23.

59 Watts NB, Harsi ST, Genant HK, et al. Intermittent cyclical etidronate treatment of postmenopausal osteoporosis. N Engl f Med 1990;323: 73-9.

60 Geddes AD, DSouza SM, Ebetin FH, et al. Bisphosphonates: structureactivity relationships and therapeutic implications. In: Heersche JNM, Kanis JA, eds. Bone and mineral. Volume 8. Oxford: Elsevier Science, 1994: 265-306.

61 Caggiano TJ, Zask A, Bex F. Recent advances in bone metabolism and osteoporosis research. In: Annual reports in medicina

62 Frith JC York: Academic Press, 1991: 201-10. en adenosine (5-( $\beta$-dichloromethylene) triphosphate by mammalian cells adenosine (5-( $\beta$-dichlomethylene) triphosphate by mammalian cells

63 Luckman SP, Hughes DE, Coxon FP, et al. Nitrogen containing bisphosphonates inhibit the mevalonate pathway and prevent post translational prenylation of GTP binding proteins, including Ras. f Bone Mineral Res 1998;13:581-9.

64 Monkkonen J, Simila J, Rogers MJ. Effects of tiludronate and ibandronate on proinflammatory cytokine and nitric oxide secretion from macrophages. Bone 1998;22(Suppl B19).

65 Luengo M, Picado C, Rio LD, et al. Vertebral fractures in steroid dependent asthma and involutional osteoporosis: a comparative study. Thorax 1991;46:803-6. 